\title{
Visual persistence from flickered and flashed gratings: Methodological considerations
}

\author{
GERALD M. LONG \\ Villanova University, Villanova, Pennsylvania \\ and \\ BARBARA SAKITT \\ Massachusetts Institute of Technology, Cambridge, Massachusetts

\begin{abstract}
Four brief experiments examine the relationships between estimates of visual persistence and spatial frequency on several currently popular tasks of persistence. Parallels between the present finding and the widely discrepant results in the persistence literature are noted. The roles of identifiably different processes underlying the various persistence tasks are suggested.
\end{abstract}

Numerous studies in the vision literature have concerned themselves with the temporal tuning (e.g., latency, duration) of the perceptual response for grating stimuli. For example, Harwerth and Levi (1978) have examined the effect of increasing spatial frequency on reaction time (RT) to the onset of a grating target. Parker (1980), using a RT task, and Long and Gildea (1981), using a probe adjustment procedure, have determined the latency for perceived offset of a brief target as a function of its spatial frequency. Other investigators, concerned with total perceived duration, have instructed observers to adjust the probe to coincide with the end of any trace of the brief grating (e.g., Long \& Beaton, 1980). Still others have flickered a constant-duration grating at various rates; and from observers' responses of "continuous" or "intermittent" at each flicker rate, the phenomenal durations of targets varying in spatial frequency have been estimated (e.g., Meyer \& Maguire, 1981).

The theoretical rationale for most of these studies has been to isolate and identify the temporal response properties of the transient and sustained channels within the visual system (Regan, 1982). However, to a significant extent, the findings from these studies frequently do not agree. High-spatial-frequency targets have been claimed to persist more (e.g., Meyer \& Maguire, 1977) and less (e.g., Long \& Sakitt, 1981) than low-spatial-frequency targets. Similar discrepant results can be found concerning the effects of varying the luminance or duration of the target grating (e.g., Bowling \& Lovegrove, 1980; Long \& Sakitt, 1981).

Given such empirical contradictions, many investigators have logically focused on the issue of the equivalence of the experimental procedures employed. For

This research was supported by National Eye Institute Grant 1 R01 EY04485-01A1 to the first author. Requests for reprints should be addressed to Gerald M. Long, Department of Psychology, Villanova University, Villanova, Pennsylvania 19085. example, the validity of the flickering task of persistence has been questioned on the grounds that it may have more in common with a critical-flicker-frequency (CFF) task of perceptual fusion than with a persistence task (e.g., Dick, 1974; Sakitt, 1976). An alternate approach to the empirical confusion has been to propose that different "types" of persistence may exist (Hawkins \& Shulman, 1979; Long, 1979). In this view, certain procedures may access the physiological correlate of perceived offset of a brief target, and others may access the physiological correlate of the total fading trace of a brief target. The value of this proposed dichotomy rests in the opposite empirical relationships exhibited by the two "types" of persistence with various stimulus manipulations (e.g., Long \& Gildea, 1981).

The present work represents a logical extension of these previous studies that have argued that the various persistence tasks employed in the current literature may not be assessing a single process or property of the human visual system. The specific aim of the research reported here was to determine the similarities and differences in the effects of varying spatial frequency on tasks commonly used to assess the temporal response properties of the visual system.

\section{EXPERIMENT 1: QUASI-FLICKER TASK}

\section{Method}

Subjects. Two graduate students served as volunteer observers in four 45-min experimental sessions each.

Apparatus and Stimuli. All background and target stimuli were presented in a three-channel Iconix tachistoscope. The two target stimuli consisted of a high-contrast 1.0 -cycle/deg and a 7.5-cycle/deg square-wave grating that completely filled the $7.5 \mathrm{x}$ $5.5 \mathrm{deg}$ target field. The grating targets and blank background fields were of constant space-averaged luminance of $5 \mathrm{fL}$ $\left(17.1 \mathrm{~cd} / \mathrm{m}^{2}\right)$. Target duration was maintained at $50 \mathrm{msec}$. The white background field contained a small fixation "plus" located centrally.

Procedure. The observers fixated the background field and, 


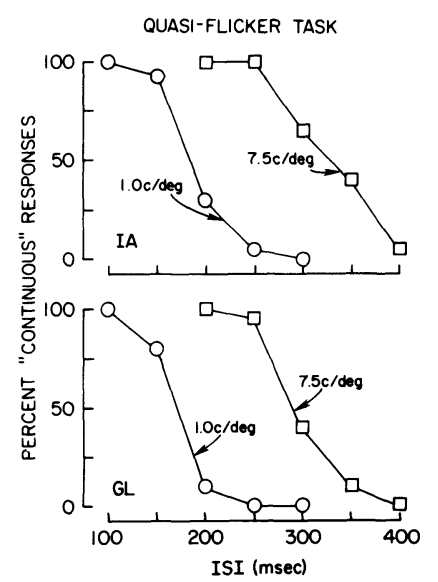

Figure 1. Percent "continuous" responses on the quasi-flicker task for two observers as a function of ISI (i.e., duration of blank field) between flickered gratings. Target gratings were either 1.0 or 7.5 cycles/deg (Experiment 1)

when ready, initiated a trial by depressing a button. As in the procedure employed by Meyer and Maguire (1977), a single trial consisted of a 50-msec grating followed by a variable-duration blank interval followed by the 50-msec grating, etc., for several (5-7) repetitions. The observer simply reported whether the grating completely disappeared during the blank period between target repetitions. For five different durations of the blank period (see Results), the observer reported whether the grating pattern was "continuous" or "intermittent." For each of the two target gratings, 40 judgments per duration of the blank period were made. These judgments were made in eight blocks of 25 trials [5 per interstimulus interval (ISI)] presented over four experimental sessions. In each session, the observer received 5 blocked trials for each grating at each of the five ISIs in a random sequence.

\section{Results}

The results for the two observers are shown in Figure 1. The abscissa represents the durations of the blank field that were presented, and the ordinate represents the percentage of "continuous" reports made by the observer at each blank-field duration. In direct replication of Meyer and Maguire (1977), Bowling and Lovegrove (1980), and others, much longer durations of the blank field are possible for reports of "continuous" with the high-spatial-frequency target than with the low-spatialfrequency target. A common interpretation of this result is in terms of "increased sustained channel mediation" (Meyer \& Maguire, 1977, p. 524) as spatial frequency is increased. This interpretation is consistent with the response characteristics typically ascribed to the transient and sustained channels in the visual system. The former channels are generally conceptualized as lowacuity/fast-responding channels, the latter as high-acuity/ slow-responding channels (see Braddick, Campbell, \& Atkinson, 1978).

\section{EXPERIMENT 2: OFFSET TASK}

\section{Method}

Subjects. Two male observers, a graduate student who had participated in Experiment 1 and an undergraduate who was paid for his participation, served as the subjects in four 45 -min experimental sessions each.

Apparatus and Stimuli. The apparatus and stimuli that had been used in Experiment 1 were again used. The only addition was a 10-msec "click," whose duration and time of occurrence after the offset of the 50-msec target grating were controlled by the Iconix timer.

Procedure. On each trial, the experimenter adjusted the ISI between the offset of the grating and the onset of the auditory probe ("click") to one of five possible values that spanned a 200 -msec range (see Results). Following a verbal "ready" signal, the observer depressed a button that initiated the target stimulus after a 50-msec delay. On each trial, the observer's task was to report whether the probe occurred "early" or "late" with respect to the perceived offset of the brief target grating. He was explicitly instructed to attend to the phenomenal offset of the target as distinct from the gradually fading trace of the target.

In a single experimental session, each target grating (1.0 or 7.5 cycles/deg) was presented in two blocks of trials. As in Experiment 1, a trial block consisted of five presentations at each of the five randomly ordered ISI values between target offset and the probe. Hence, over the four sessions, each ISI condition was presented 40 times for each of the two target gratings.

\section{Results and Discussion}

The results for the two observers are shown in Figure 2. The precentage of "early" responses by the two observers for each of the target gratings is shown as a function of the ISI between target offset and the probe. For both observers, the curve for the high-spatialfrequency target is shifted to the right by about $100 \mathrm{msec}$. The similarity of Figures 1 and 2, along with the fact that previous research has shown both persistence tasks to exhibit an inverse relationship with target luminance and target duration (e.g., Bowling \& Lovegrove, 1980; Meyer \& Maguire, 1981), suggests that the same process(es) may underlie the two experimental procedures employed in Experiments 1 and 2.

\section{EXPERIMENT 3: TOTAL PERSISTENCE}

\section{Method}

Subjects. The same two male observers employed in Experiment 1 again served as the subjects.

Apparatus and Stimuli. The apparatus and stimuli used in Experiment 2 were employed.

Procedure. Whereas in Experiment 2 the observers reported whether the auditory probe was "early" or "late" with respect to target offset, in Experiment 3 they reported whether the probe was "early" or "late" with respect to any trace of the brief target. As Corwin, Volpe, \& Tyler (1976) and Sakitt and Long (1979) have reported, observers have little difficulty making this distinction between the phenomenal offset of a target and the end of any trace of the target.

\section{Results and Discussion}

The results for the two observers are shown in Figure 3. The percentage of "early" responses is plotted as a function of ISI for each of the two target gratings. There are two notable differences between these results and those obtained in Experiment 2. First, the ISI range in Figure 3 has been greatly expanded to accommodate the observers' reports. That is, total persistence far outlasts the latency for perceived offset of the target gratings. Hence, whatever processes underlie the results in the present experiment, they are much slower than those 


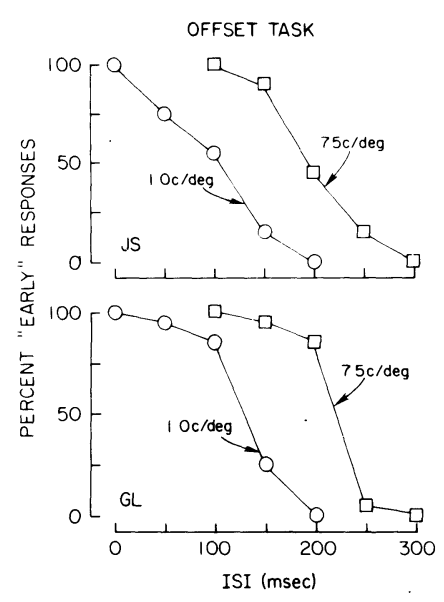

Figure 2. Percentage of "early" responses on the offset task for two observers as a function of ISI between target offset and probe onset. Target gratings were either 1.0 or 7.5 cycles/deg. (Experiment 2)

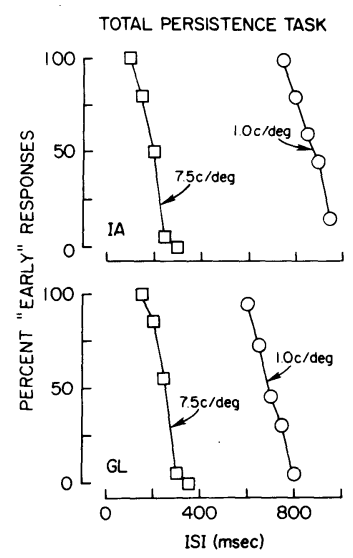

Figure 3. Percentage of "early" responses on the totalpersistence task for two observers as a function of ISI between target offset and probe onset. Target gratings were either 1.0 or 7.5 cycles/deg. (Experiment 3)

responsible for the results in the previous two experiments. Second, the low-frequency target persists much longer than the high-frequency target. For both observers, the curve for the 1.0-cycle/deg grating is shifted several hundred milliseconds to the right of the curve for the 7.5-cycle/deg grating. Hence, it is clear from a comparison of Figures 2 and 3 that perceived offset of a brief target is not equivalent to the end of any persistence from that target and that the two phenomenal events exhibit a very different relationship with spatial frequency.

\section{EXPERIMENT 4: CFF vs. QUASI-FLICKER}

As noted in the introduction, several researchers have commented on the general similarity of the quasiflicker task of persistence (Experiment 1) and the stan- dard CFF task of perceptual fusion (e.g., Dick, 1974; Sakitt, 1976). In the former task, the observer seeks total fusion of the intermittent stimulus; in the latter task, the observer seeks a lack of disappearance of the intermittent stimulus. Whether fundamentally different processes or just different criteria for responding are involved across the tasks is unknown. Moreover, both tasks exhibit faster processing (i.e., briefer ISIs) as target luminance is increased (e.g., Haber \& Standing, 1969; Hecht \& Smith, 1936). Experiment 4 attempted to address this issue of the comparability of the CFF and quasi-flicker tasks. For the same observers, the effect of varying spatial frequency was assessed on a CFF and a quasi-flicker task under identical stimulus and procedural conditions.

\section{Method}

Subjects. Two male observers, the same graduate student who had participated in the first three experiments and an undergraduate who was paid for participation, served as the subjects in four 45-min sessions.

Apparatus and Stimuli. The apparatus and stimulus conditions employed in Experiment 1 were again used.

Procedure. In two of the experimental sessions (randomly selected), observers were instructed to employ a strict CFF criterion such that the perception of any hint of flicker in the target was to be reported as "noncontinuous." For each of the two 50-msec gratings, the interval between target flashes was varied over several values (see Results). Each ISI value was presented in 20 total trials over the two sessions. In the other two experimental sessions, the same quasi-flicker procedure described in Experiment 1 was employed. The observers were instructed to report if the target grating at any point disappeared in the blank interval between target flashes. The perception of flicker per se was irrelevant.

\section{Results and Discussion}

The results for the two observers under both instructional sets are shown in Figure 4. For both observers, the results with the quasi-flicker instructions replicate the findings of Experiment 1. The particular interest of the present study, however, concerns the results obtained with the CFF instructions and their similarity to those obtained with the quasi-flicker task of persistence. It can be seen from Figure 4 that, for both observers, the two CFF curves are shifted to the left of the two quasi-flicker curves. This is to be expected because of the stringent instructions given to observers on the CFF task to report any perceptual flicker as "noncontinuous." However, note too that the effect of varying spatial frequency on the CFF task is in the same direction as that obtained on the quasi-flicker task of persistence. The 7.5-cycle/deg target permits reports of both pattern continuity and complete perceptual fusion at longer ISIs than does the 1.0-cycle/deg target. This would be consistent with the claim that the same process(es) underlie both tasks but that a response-criterion difference across instructional demands produces differences in the absolute ISI levels obtained. The potential importance of such a claim rests on the generally accepted view that many mechanisms interact to produce flicker phenomena. This in turn raises the question of the validity of the quasi-flicker task as an unambiguous measure of persis- 


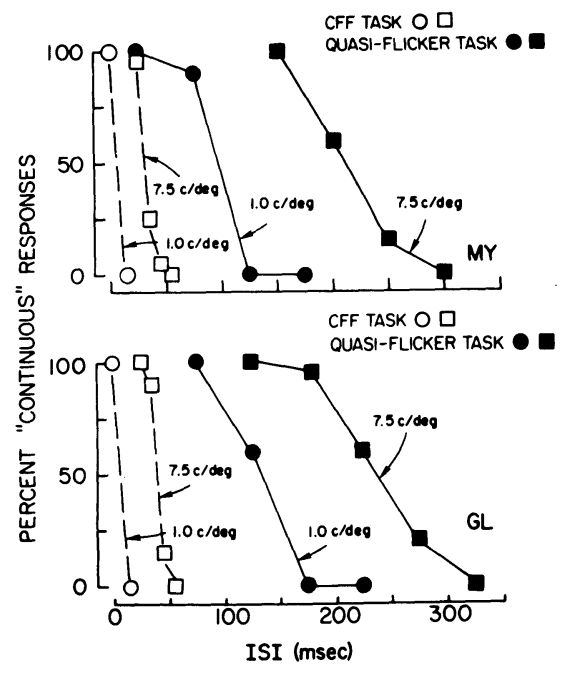

Figure 4. Percentage of "continuous" responses on the CFF task and the quasi-flicker task for two observers as a function of ISI (i.e., duration of blank field) between flickered gratings. Target gratings were either 1.0 or 7.5 cycles/deg. (Experiment 4)

tence or, at least, its comparability with other traditional persistence tasks (see also Long \& Sakitt, 1981).

\section{GENERAL CONCLUSION}

The results reported here clearly demonstrate powerful differences across several currently popular measures of visual persistence. Moreover, these differences cannot be attributed to differences in apparatus, stimulus conditions, or observers, which were identical across the tasks investigated. These findings would appear to support the recent arguments (Long, 1982) that different perceptual processes underlie some of the popular persistence tasks. This in turn is the probable basis for the apparent empirical discrepancies that plague the current persistence literature (Coltheart, 1980).

The acceptance of the present proposal that very different processes may be involved across currently popular persistence tasks indicates a need for caution in generalizing from particular empirical findings. Given multiple processes that may underlie visual persistence effects, a particular experimental task very likely assesses some subset of such processes. Recognizing this fact, future theory and research can then focus on the different processes assessed by the variety of persistence tasks as well as on the involvement of these separable persistence processes in numerous other visual phenomena, such as illusions (e.g., Walker, 1981), masking (e.g., Breitmeyer, 1980), reading disabilities (e.g., Badcock \& Lovegrove, 1981), age changes in perception (e.g., Kline \& Schieber, 1981), information processing (e.g., Coltheart, 1980), and more.

\section{REFERENCES}

Badcock, D., \& Lovegrove, W. The effects of contrast, stimulus duration, and spatial frequency on visible persistence in normal and specifically disabled readers. Journal of Experimental Psychology: Human Perception and Performance, 1981, 7, 495-505.
Bowling, A., \& Lovegrove, W. The effect of stimulus duration on the persistence of gratings. Perception \& Psychophysics, 1980, 27, 574-578.

Braddick, O. J., Campbell, F. W., \& Atxinson, J. Channels in vision: Basic aspects. In R. Held, H. W. Leibowitz, \& H. L. Teuber (Eds.), Handbook of sensory physiology (Vol. 8): Perception. Berlin: Springer, 1978.

BreitMeyer, B. G. Unmasking visual masking: A look at the "why" behind the veil of the "how." Psychological Review, $1980,87,52-69$.

Coltheart, M. Iconic memory and visible persistence. Perception \& Psychophysics, 1980, 27, 183-228.

Corwin, T. R., Volpe, L. C., \& TYLER, C. W. Images and afterimages of sinusoidal gratings. Vision Research, 1976, 16, 345-350.

Dick, A. O. Iconic memory and its relation to perceptual processing and other memory mechanisms. Perception \& Psychophysics, 1974, 16, 575-596.

Haber, R. N., \& Standing, L. Direct measures of short term visual storage. Quarterly Journal of Experimental Psychology, $1969,21,43-54$.

Harwerth, R. S., \& Levi, D. M. Reaction time as a measure of suprathreshold grating detection. Vision Research, 1978, 18, 1579-1586.

Hawkins, H. L., \& Shulman, G. L. Two definitions of persistence in visual perception. Perception \& Psychophysics, 1979, 25, 348-350.

Hеснт, S., \& Sмiтн, E. L. Intermittent stimulation by light. VI. Area and the relation between critical frequency and intensity. Journal of General Physiology, 1936, 19, 979-991.

KLINE, D. W., \& Schie BE R, F. Visual aging: A transient/sustained shift? Perception \& Psychophysics, 1981, 29, 181-182.

Long, G. M. Comment on Hawkins and Shulman's Type I and Type II visual persistence. Perception \& Psychophysics, 1979, 26, 412-414.

Long, G. M. Persisting problems in persistence: A reply to Bowling and Lovegrove. Perception \& Psychophysics, 1982, 32, 192-194.

Lona, G. M., \& Beaton, R. J. The effects of spatial frequency and target type on perceived duration. Perception \& Psychophysics, 1980, 28, 413-421.

Long, G. M., \& GildeA, T. J. Spatial frequency and the latency for perceived of fset. Vision Research, 1981, 21, 1395-1399.

Long, G. M. \& SAKITT, B. Differences between flicker and nonflicker persistence tasks: The effects of luminance and the number of cycles in a grating target. Vision Research, 1981, 21, 1387-1393.

Meyer, G. E., \& Maguire, W. M. Spatial frequency and the mediation of short term visual storage. Science, 1977, 198, 524-525.

MEYer, G. E., \& MAGuire, W. M. Effects of spatial-frequency specific adaptation and target duration on visual persistence. Journal of Experimental Psychology: Human Perception and Performance, 1981, 7, 151-156.

Parker, D. M. Simple reaction times to the onset, of fset and contrast reversal of sinusoidal grating stimuli. Perception \& Psychophysics, 1980, 38, 365-368.

REGAN, D. Visual information channeling in normal and disordered vision. Psychological Review, 1982, 89, $407-444$.

Sakitr, B. Iconic memory. Psychological Review, 1976, 83, 257-276.

Sakitr, B., \& Long, G. M. Cones determine subjective offset of a stimulus but rods determine total persistence. Vision Research, $1979,19,1439-1441$.

WAlker, J. T. A new dynamic visual illusion: The bending hourglass. Perception \& Psychophysics, 1981, 30, 455-458.

(Revision received for publication September 22, 1983.) 